\title{
SUBSHIFTS, ROTATIONS AND THE SPECIFICATION PROPERTY
}

\author{
Marcin Mazur — Piotr Oprocha
}

\begin{abstract}
Let $X=\Sigma_{2}$ and let $F: X \times \mathbb{S}^{1} \rightarrow X \times \mathbb{S}^{1}$ be a map given by $F(x, t)=\left(\sigma(x), R_{x_{0}}(t)\right)$,

where $\left(\Sigma_{2}, \sigma\right)$ denotes the full shift over the alphabet $\{0,1\}$ while $R_{0}, R_{1}$ are the rotations of the unit circle $\mathbb{S}^{1}$ by the angles $r_{0}$ and $r_{1}$, respectivelly. It was recently proved by $\mathrm{X}$. Wu and $\mathrm{G}$. Chen that if $r_{0}$ and $r_{1}$ are irrational, then the system $\left(X \times \mathbb{S}^{1}, F\right)$ has an uncountable distributionally $\delta$-scrambled set $S_{\delta}$ for every positive $\delta \leq \operatorname{diam} X \times \mathbb{S}^{1}=1$. Moreover, each point in $S_{\delta}$ is recurrent but not weakly almost periodic (this answeres a question from [Wang et al., Ann. Polon. Math. 82 (2003), 265-272]).

We generalize the above result by proving that if $r_{0}-r_{1} \in \mathbb{R} \backslash \mathbb{Q}$ and $X \subset \Sigma_{2}$ is a nontrivial subshift with the specification property, then the system $\left(X \times \mathbb{S}^{1}, F\right)$ also has the specification property. As a consequence, there exist a constant $\delta>0$ and a dense Mycielski distributionally $\delta$-scrambled set for $\left(X \times \mathbb{S}^{1}, F\right)$, in which each point is recurrent but not weakly almost periodic.
\end{abstract}

\section{Introduction}

In the field of the qualitative theory of dynamical systems, the objects of a special interest are dynamical systems that exhibit any kind of chaotic behavior. Probably the most famous definition of chaos comes from Devaney [6] and involves such notions as transitivity or sensitivity. In this paper we investigate

2010 Mathematics Subject Classification. Primary: 37B05, 37B10, 37B20; Secondary: $54 \mathrm{H} 20$.

Key words and phrases. Distributional chaos, scrambled set, specification property, symbolic dynamics, circle rotation. 
another concept that is known as a distributional chaos and was introduced by Schweizer and Smítal in [18] (under the name "strong chaos"). Roughly speaking, it relates to the complexity of dynamics with a distribution function of average distances between trajectories of two points (see the next section for a definition).

The specification property was first introduced by Bowen [4], and it is one of the strongest mixing properties. On the other hand, rotations of the circle are examples of the simplest dynamical systems - they are, in fact, isometries and their dynamics is, in some sense, rigid. However, it is known that skew product systems involving irrational rotations can lead to surprising examples. Among the most interesting results in this direction are the constructions from [9], where irrational rotations on tori are used as a tool to construct a weakly mixing, minimal and uniformly rigid skew product system. This shows that simple rotations, when properly controlled (or sufficiently randomized, depending on point of view), may lead to chaotic systems with mixing properties. In our work we are going to show that a kind of complicated dynamics, involving the specification property, can arise as a consequence of a proper control (in the sense of a skew product) of two (appropriately chosen) rotations. This result seems to be somewhat surprising, since on each fiber (i.e., the unit circle) such dynamics is, in fact, rigid, in the sense that all points never change their relative positions.

The origins of our model can be found in a paper by Falcó [8], who in fact was inspired by much earlier paper by Afraimovich and Shilnikov, published in 1974 [1]. In [8], Falcó was interested in describing the set of periods for the skew product system in the form of (3.1), defined by $N$ rotations and a full two-sided shift over $N$ symbols. The present paper is motivated by the works of Wang et al. [19] and Wu and Chen [21]. In [19] there is a question concerning the existence of an uncountable distributionally scrambled set $S$ (a dynamical system with a distributional chaos) such that each element of $S$ is a recurrent point. Wang et. al. formulated a sufficient condition for the existence of such a set $S$ (moreover, the chaotic set $S$ they obtained by this condition did not contain almost periodic points). They also presented an example of a class of "shift directed alternating systems" of irrational rotations of the unit circle (see Section 3 of the present paper for precise statement), for which they were not able to verify the introduced condition and hence failed to prove in this case the presence of a distributional chaos. The discussion was then continued in [21], where it was shown that the systems considered in [19] actually contain an uncountable distributionally scrambled set, in which each point is recurrent but not weakly almost periodic.

In our paper we use the concept of the specification property in order to formulate another global condition, guaranteeing the presence of a distributional 